\title{
Qualités bouchères de lapereaux issus d'un croisement diallèle de 3 souches : interaction du type génétique et de la taille de portée d'origine
}

\author{
JM Brun ${ }^{1}$, J Ouhayoun ${ }^{2}$ \\ 1 INRA, station d'amélioration génétique des animaux; \\ 2 INRA, station de recherches cunicoles, BP 27, 31326 Castanet-Tolosan cedex
}

(Reçu le 6 janvier 1992; accepté le 14 décembre 1993)

\begin{abstract}
Résumé - L'effet de la taille de portée et du type génétique sur la croissance entre 30 et 79 j de 605 lapereaux et les qualités bouchères de 300 d'entre eux a été étudié dans un plan de croisement diallèle entre 3 souches de l'INRA, les souches 1066 et 1077, sélectionnées depuis 13 générations sur la taille de portée et la souche 9077, témoin de la souche 1077. À l'inverse de la souche 1066, la souche 9077 transmet des effets directs favorables sur la vitesse de croissance et le poids à l'abattage mais défavorables sur les qualités bouchères. La souche 1077 exerce des effets matemels défavorables sur la vitesse de croissance et le poids de carcasse ajusté pour le poids vif (indicateur du rendement en carcasse) et des effets directs qui abaissent l'adiposité des carcasses. Des effets d'hétérosis significatifs sont observés sur le poids au sevrage $(5 \%)$ dans tous les croisements, sur le rendement en carcasse $(2 \%)$ dans les croisements $1077 \times 9077$ et $9077 \times 1066$ et sur la vitesse de croissance $(7 \%)$ dans le croisement $1066 \times 1077$. La sélection sur la prolificité dans la souche 1077 s'est accompagnée d'une diminution de la vitesse de croissance et de l'adiposité des carcasses, due aux effets génétiques directs, et d'une diminution de la proportion des membres postérieurs, due aux effets génétiques maternels. Des interactions significatives sont trouvées entre les types génétiques du père ou de la mère du lapereau et sa taille de portée d'origine. Les lapereaux de père 1077 ne subissent pas d'effet dépressif de la taille de portée sur le poids à 30 et à $79 \mathrm{j}$. Les lapereaux de père ou de mère 1066, dont le poids à 30 j est diminué par une taille de portée élevée, ne bénéficient pas d'une croissance compensatrice.
\end{abstract}

lapin / génétique / croisement / croissance / qualités bouchères

Summary - Effects of litter size and genetic type on growth and carcass traits of rabbit in a 3strain diallel cross. The effects of litter size and genetic type on growth between 30 and $79 d$ of 605 rabbits and carcass traits of 300 rabbits were analyzed in a diallelle cross between 3 INRA strains. Strains 1066 and 1077 were both selected on litter size for 13 generations and the 9077 strain was used as a control line for 1077. Inversely to strain 1066, strain 9077 had favourable direct effects on growth rate and slaughter weight but unfavourable ones on carcass quality. Strain 1077 exerted unfavourable maternal effects on growth rate, carcass weight adjusted for live weight (estimator of slaughter yield) and decreased carcass fatness through direct effects. Significant heterosis was found on 30-d body weight (5\%) in all crosses, on slaughter yield (2\%) in $1077 \times 9077$ and $9077 \times 1066$ crosses and on 
growth rate (7\%) in the cross between strains 1066 and 1077. Selection on prolificacy resulted in a decrease in growth rate and carcass fatness, due to direct genetic effects, and in hindleg fraction of the carcass, due to maternal genetic effects. Significant interactions were found between sire or dam genetic type and litter size from which the rabbits originate. Rabbits from 1077 sires did not suffer any reduction in 30- and 79-d weight due to larger litter size. Rabbits from 1066 sires or dams, whose 30-d body weight was decreased by a larger litter size, did not exhibit any compensatory growth.

\section{rabbit / genetics / crossbreeding / growth / carcass traits}

\section{INTRODUCTION}

En production de viande de lapin, le croisement de souches spécialisées permet de bénéficier de la complémentarité entre la voie femelle, à bonne aptitude reproductive, et la voie mâle, à bonne aptitude bouchère. Si les souches femelles sont généralement sélectionnées sur la prolificité, il ne faut pas négliger le fait qu'elles contribuent, au moins pour moitié, aux caractéristiques bouchères de leurs descendants. Cela justifie d'estimer les effets génétiques sur les qualités bouchères que ces souches sont susceptibles de transmettre en croisement. C'est ce qui a été réalisé une première fois en 1987 (Brun et Ouhayoun, 1989), sur 3 souches de lapins de l'INRA, dont 2, sélectionnées sur la prolificité, sont à l'origine de la femelle métisse parentale INRA 1067. Le présent article concerne l'analyse d'une répétition, portant sur un effectif plus important.

\section{MATÉRIEL ET MÉTHODES}

\section{Animaux et conditions expérimentales}

Les souches utilisées sont les souches INRA 1066, d'origine raciale Californienne, 1077 et 9077, d'origine raciale Néo-Zélandaise Blanche. Les 2 premières sont sélectionnées sur la prolificité. La troisième est un témoin non sélectionné. En 13 générations de sélection, la taille de portée a progressé de 0,8 lapereau à la naissance et de 0,6 lapereau au sevrage dans la souche 1077, par rapport à la souche 9077 (Brun, 1990). Les trois souches sont de format adulte moyen. Cependant, la souche 9077 , dont le poids adulte est de l'ordre de $4,1 \mathrm{~kg}$, est plus lourde d'environ $8 \%$ que les 2 autres.

La présente étude concerne 605 lapereaux des 2 sexes issus d'un plan de croisement diallèle entre les 3 souches. Chacune des souches est représentée par 10 mâles et 30 femelles. Les lapereaux sont nés en juin et juillet 1988. Ils proviennent de portées de rangs $2(14 \%)$ et $3(86 \%)$ et d'effectif au sevrage supérieur à 3 . II n'y a eu ni retrait ni adoption de lapereaux à la naissance. Les lapereaux ont été élevés selon les conditions habituelles des élevages expérimentaux de la station d'amélioration génétique des animaux : sevrage à $30 \mathrm{j}$, engraissement en cages collectives disposées en flat-deck (16 lapereaux par $\mathrm{m}^{2}$ ), alimentation à volonté (aliment standard commercial).

Un échantillon de 300 lapereaux mâles a été abattu à l'âge de $79 \mathrm{j}$. lls ont été saignés par section de la veine jugulaire et de l'artère carotide après électroanesthésie $(90 \mathrm{~V}, 2 \mathrm{~s})$. Les carcasses ont été réfrigérées $\left(+2^{\circ} \mathrm{C}\right)$ pendant $22 \mathrm{~h}$. Elles ont été analysées conformément aux recommandations de Blasco et al (1993). La répartition des lapereaux contrôlés sur la croissance et des lapereaux abattus, par souche de père et de mère, est donnée au tableau $\mathrm{l}$.

\section{Caractères contrôlés}

- Taille de portée au sevrage.

- Poids vif (g) à $30 \mathrm{j}$ (sevrage).

- Poids vif (g) à $79 \mathrm{j}$ (abattage).

- Gain de poids $(\mathrm{g})$ entre 30 et $79 \mathrm{j}$. 
Tableau I. Nombre de lapereaux contrôlés sur la croissance $\left({ }^{a}\right)$ et sur les qualités bouchères $\left({ }^{b}\right)$ par type génétique de père, de mère et de lapereau.

\begin{tabular}{lcccc}
\hline \multirow{2}{*}{ Type génétique de père } & \multicolumn{3}{c}{ Type génétique de mère } & Total \\
\cline { 2 - 4 } & 9077 & 1066 & 1077 & \\
\hline & & & & \\
9077 & $73^{\mathrm{a}}$ & $67^{\mathrm{a}}$ & $61^{\mathrm{a}}$ & $201^{\mathrm{a}}$ \\
& $42^{\mathrm{b}}$ & $29^{\mathrm{b}}$ & $32^{\mathrm{b}}$ & $103^{\mathrm{b}}$ \\
1066 & $78^{\mathrm{a}}$ & $59^{\mathrm{a}}$ & $76^{\mathrm{a}}$ & $213^{\mathrm{a}}$ \\
& $36^{\mathrm{b}}$ & $32^{\mathrm{b}}$ & $36^{\mathrm{b}}$ & $104^{\mathrm{b}}$ \\
1077 & $71^{\mathrm{a}}$ & $48^{\mathrm{a}}$ & $72^{\mathrm{a}}$ & $191^{\mathrm{a}}$ \\
& $40^{\mathrm{b}}$ & $19^{\mathrm{b}}$ & $34^{\mathrm{b}}$ & $93^{\mathrm{b}}$ \\
\multirow{3}{*}{ Total } & & & & $605^{\mathrm{a}}$ \\
& $222^{\mathrm{a}}$ & $174^{\mathrm{a}}$ & $209^{\mathrm{a}}$ & $300^{\mathrm{b}}$ \\
\hline
\end{tabular}

- Poids de carcasse commerciale.

- Poids d'un membre postérieur.

- Poids de la fraction comestible d'un membre postérieur.

- Poids du tissu adipeux périrénal.

\section{Analyses statistiques}

Les poids à âge-type et le gain de poids ont été traités selon un modèle d'analyse de variance, avec les effets du type génétique du père du lapereau, de sa mère et de l'interaction de ces 2 facteurs; la taille de portée d'origine au sevrage du lapereau a été introduite comme covariable selon un modèle dit "d'hétérogénéité des pentes" (SAS/Stat, 1990), où les coefficients de régression peuvent varier selon le type génétique du père ou celui de la mère ; le test de cette hétérogénéité est indicatif de l'existence d'interaction entre la taille de portée et le type génétique du père ou de la mère du lapereau.

Le poids de la carcasse commerciale, le poids du membre postérieur et du gras périrénal, et enfin le poids de la partie comestible du membre postérieur ont été analysés avec le modèle précédent incluant une covariable supplémentaire : le poids vif à l'abattage, le poids de la carcasse ou le poids du membre postérieur. Ces variables ajustées représentent, respectivement, le rendement en carcasse, la proportion du membre postérieur et du gras périrénal dans la carcasse et la fraction comestible du membre postérieur. Les effets du sexe du lapereau et du numéro de la portée d'origine, trouvés non significatifs dans une analyse préliminaire, n'ont pas été inclus dans le modèle.

Une analyse supplémentaire du poids à $30 \mathrm{j}$ a été réalisée selon un modèle où la taille de portée est considérée comme effet fixé et non comme covariable afin de déterminer le poids à $30 \mathrm{j}$ des lapereaux dans 2 modalités de la taille de portée : de 4 à 8 et de 9 à 13 lapereaux.

\section{Analyse génétique}

Le modèle de Dickerson (1969) a été utilisé pour estimer les paramètres génétiques du croisement : les effets génétiques additifs directs $\left(\mathrm{g}^{\prime}\right)$, maternels plus grand-maternels $\left(\mathrm{g}^{\mathrm{M}}\right)$, ainsi que les effets d'hétérosis directs $\left(\mathrm{h}^{\prime}\right)$. Ces paramètres ont été calculés à partir des estimées des valeurs moyennes des types génétiques de lapereaux selon la méthode décrite par Brun et Rouvier (1984). Comme ces dernières, les paramètres du croisement sont ajustés pour la taille de portée au sevrage.

Ils n'ont été estimés que pour les caractères soumis à un effet du type de père, de mère ou d'interaction père $x$ mère. La signification des paramètres génétiques a été testée par la méthode de Student. 


\section{RÉSULTATS}

\section{Effets du type génétique du père et de la mère (tableaux II et III)}

Le modèle d'analyse utilisé explique, selon le caractère, de 14 à $95 \%$ de la variabilité. Les caractères de croissance sont influencés par le type génétique du père, de la mère et par l'interaction de ces 2 facteurs, à l'exception du gain de poids, qui n'est pas influencé par le type génétique du père. Le poids du membre postérieur à poids de carcasse constant est influencé par les types génétiques du père et de la mère. Le poids de la carcasse ajusté pour le poids vif est influencé par l'interaction de ces 2 facteurs.

En présence d'interaction entre les types génétiques du père et de la mère sur les poids à âge-type et le gain de poids, il est difficile de commenter les effets principaux.
Toutefois il semble que le classement des types génétiques de mère soit relativement constant quel que soit le type génétique de père, alors que l'inverse n'est pas vérifié. Pour les poids à $30 \mathrm{j}$ et à $79 \mathrm{j}$, les types de mère se classent ainsi : $9077>1066=$ 1077. Ce classement reste valable pour le gain de poids, sauf avec les pères de souche 1077. Le poids de carcasse ajusté pour le poids vif ne dépend que de l'interaction des types de père et de mère. Le poids du membre postérieur à poids de carcasse constant est influencé par le type du père et de la mère : utilisée comme père ou comme mère, la souche 1066 est la mieux classée. Aucun effet génétique n'apparaît significatif sur la fraction comestible du membre postérieur (poids des tissus comestibles ajusté). En ce qui concerne l'adiposité de la carcasse (poids du tissu adipeux périrénal ajusté), les produits de père 9077 présentent toujours les valeurs les plus fortes.

Tableau II. Signification des effets dans l'analyse de variance des caractères de croissance et de carcasse.

\begin{tabular}{|c|c|c|c|c|c|c|c|c|}
\hline & \multirow[t]{2}{*}{$T G P$} & \multirow[t]{2}{*}{$T G M$} & \multirow[t]{2}{*}{$\begin{array}{r}\text { Interaction } \\
T G P \times T G M\end{array}$} & \multirow[t]{2}{*}{ TPO } & \multicolumn{2}{|c|}{$\begin{array}{l}\text { Interactions de } \\
\text { TPO avec }\end{array}$} & \multirow[t]{2}{*}{$\sigma E$} & \multirow[t]{2}{*}{$R^{2}$} \\
\hline & & & & & $T G P$ & $T G M$ & & \\
\hline $\mathrm{P} 30(\mathrm{~g})$ & $* *$ & * & ** & ** & ** & NS & 93 & 0,34 \\
\hline P79 (g) & ** & ** & ** & $\star \star$ & $\star \star *$ & $\star \star \star$ & 193 & 0,19 \\
\hline $\mathrm{DP}(\mathrm{g})$ & NS & $* *$ & $* *$ & * & NS & $* *$ & 153 & 0,14 \\
\hline PCar ajusté (g) & NS & NS & $\star \star \star$ & NS & NS & $\star$ & 39,1 & 0,95 \\
\hline PMpos ajusté ( $\mathrm{g}$ ) & $*$ & $*$ & NS & $*$ & $\star \star$ & NS & 5,7 & 0,94 \\
\hline PFrCo ajusté (g) & NS & NS & NS & NS & NS & NS & 6,4 & 0,91 \\
\hline PTad ajuste $(\mathrm{g})$ & 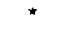 & NS & NS & * & ${ }^{* \star}$ & NS & 5,1 & 0,56 \\
\hline
\end{tabular}

NS, *, "* : non significatif $(P>0,05)$, significatif $(P<0,05)$, hautement significatif $(P<0,01) ; \sigma \mathrm{E}, \mathrm{R}^{2}$ : écart-type résiduel, coefficient de détermination du modèle; TGP, TGM : type génétique du père, de la mère; TPO : taille de la portée d'origine du lapereau, au sevrage (covariable); $P 30$ : poids vif à $30 \mathrm{j} ; \mathrm{P} 79$; poids vif à $79 \mathrm{j}$; DP : gain de poids entre 30 et $79 \mathbf{j}$; PCar ajusté : poids de carcasse commerciale ajusté pour le poids vif à $79 \mathrm{j} ;$ MPpos ajusté : poids d'un membre postérieur ajusté pour le poids de carcasse ; PFrCo ajusté : poids de la fraction comestible d'un membre postérieur ajusté pour le poids de ce dernier ; PTad ajusté : poids du tissu adipeux périrénal ajusté pour le poids de la carcasse. 
Tableau III. Croissance et qualités bouchères : moyennes par type génétique de lapereau, de père et de mère.

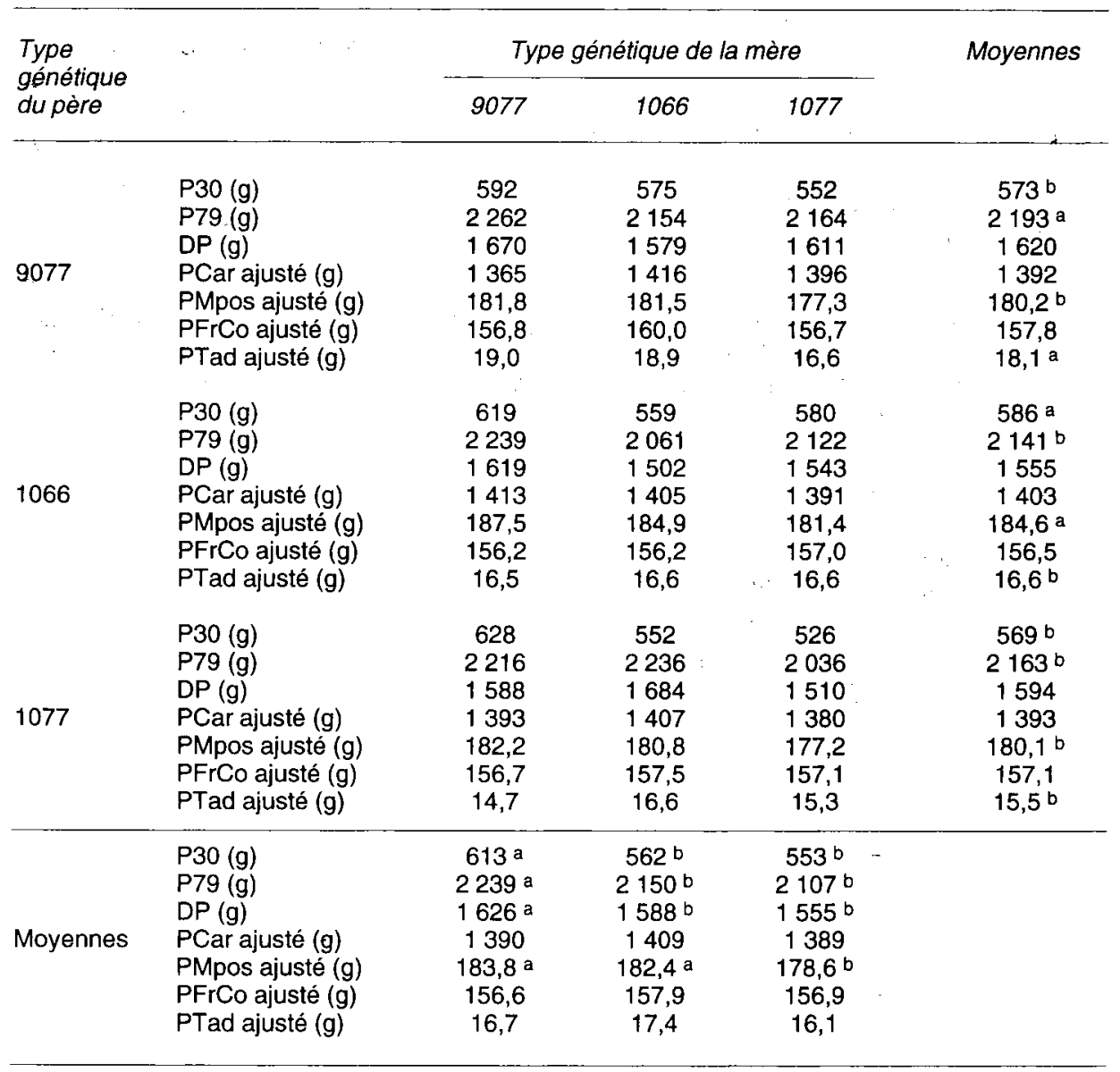

Les moyennes marginales affectées de lettres différentes sont significativement différentes $(P<0,05)$. P30 : poids vif à $30 \mathrm{j}$; P79 : poids vif à $79 \mathrm{j}$; DP : gain de poids entre 30 et $79 \mathrm{j}$; PCar ajusté : poids de carcasse commerciale ajusté pour le poids vif à 79 j ; MPpos ajusté : poids d'un membre postérieur ajusté pour le poids de carcasse : PFrCo ajusté : poids de la fraction comestible d'un membre postérieur ajusté pour le poids de ce dernier ; PTad ajusté : poids du tissu adipeux périrénal ajusté pour le poids de la carcasse.

Les 2 souches 1066 et 1077, toutes deux sélectionnées sur la taille de portée, ont des caractéristiques de croissance peu différentes mais la souche 1066 est supérieure pour le rendement en carcasse et le développement de l'arrière. La supériorité de la souche 1066 pour ces 2 caractères semble être une caractéristique raciale distinguant les origines Californienne et NéoZélandaise Blanche. La souche sélectionnée 1077 se distingue de son témoin par une vitesse de croissance, un développement relatif de l'arrière et une adiposité inférieurs. 


\section{Effets de la taille de portée d'origine et de ses interactions avec les facteurs génétiques}

L'effet de la taille de portée d'origine et de ses interactions est estimé par les coefficients de régression des variables de croissance et de carcasse sur la taille de portée. Ces coefficients sont donnés au tableau IV.

Les coefficients de régression globaux montrent qu'une augmentation de la taille de portée se traduit par une réduction du poids individuel au sevrage $(b=-27,3 \mathrm{~g} /$ unité de taille de portée) et à $79 \mathrm{j}(\mathrm{b}=-18,4)$. La réduction plus faible du poids à $79 \mathrm{j}$ résulte d'une croissance compensatrice des animaux des portées les plus nombreuses. L'augmentation de la taille de portée entraîne également une réduction de la proportion de l'arrière de la carcasse et une augmentation de l'adiposité.
Au sevrage, un effet négatif de la taille de portée sur le poids des lapereaux est observé quel que soit le type génétique de la mère. En revanche, à 79 j, seuls les lapereaux de mères 1066 sont soumis à un effet négatif de la taille de portée ; ainsi, contrairement aux produits des mères 1077 et 9077 , les produits des mères 1066 ne manifestent pas de croissance compensatrice entre 30 et $79 \mathrm{j}$. L'effet de la taille de portée sur les poids à 30 et à $79 \mathrm{j}$ dépend du type génétique du père. Sur le poids à $30 \mathrm{j}$, l'effet négatif de la taille de portée est beaucoup plus important dans les types génétiques 9077 et 1066 . Aucune différence entre types de père n'est mise en évidence sur la croissance compensatrice (absence d'interaction entre la taille de portée et le type génétique de père sur le gain de poids entre 30 et $79 \mathrm{j}$ ). Cependant, les produits des pères 1066 ne semblent pas en manifester, comme ceux des mères de même type génétique.

Tableau IV. Coefficients de régression des variables de croissance et de carcasse sur la taille de la portée d'origine du lapereau, en fonction du type génétique du père et de la mère.

\begin{tabular}{|c|c|c|c|c|c|c|c|}
\hline \multirow[t]{2}{*}{ Caractère } & \multirow[t]{2}{*}{ Global } & \multicolumn{3}{|c|}{ Type génétique de père } & \multicolumn{3}{|c|}{ Type génétique de mère } \\
\hline & & 9077 & 1066 & 1077 & 9077 & 1066 & 1077 \\
\hline P30 (g) & $-27,3$ & $-36,2 b$ & $-33,7 b$ & $-12,1^{c}$ & & & \\
\hline $\mathrm{P} 79$ (g) & $-18,4$ & $-28,0^{b}$ & $-33,1^{b}$ & 5,9 a & $-12,0$ a & $-41,0^{b}$ & $-1,0^{a}$ \\
\hline$D P(g)$ & 9,0 & - & - & - & $17,0 \mathrm{~b}$ & $-9,6$ a & $19,5^{b}$ \\
\hline PCar ajusté (g) & - & - & - & - & $-3,78$ a & $5,84 t$ & 1,26 ab \\
\hline PMpos ajusté (g) & $-0,48$ & $-1,51^{b}$ & 0,32 a & $-0,24 a b$ & - & - & - \\
\hline PFrCo ajusté (g) & - & - & - & - & - & - & - \\
\hline PTad ajusté (g) & 0,47 & $1,30^{b}$ & $-0,13 a b$ & $-0,23$ a & & & \\
\hline
\end{tabular}

Seules les valeurs correspondant à des effets significatifs ( $c$ tableau II) sont reportées dans le tableau. Intra type génétique de père ou de mère, les coefficients affectés de lettres différentes sont significativement différents $(P<0,05)$. Ceux qui sont affectés de la lettre a ne diffèrent pas de zéro. P30 : poids vif à $30 \mathrm{j}$; P79 : poids vif à $79 \mathrm{j}$; DP : gain de poids entre 30 et $79 \mathrm{j}$; PCar ajusté : poids de carcasse commerciale ajusté pour le poids vif à $79 \mathbf{j}$; MPpos ajusté : poids d'un membre postérieur ajusté pour le poids de carcasse ; PFrCo ajusté : poids de la fraction comestible d'un membre postérieur ajusté pour le poids de ce dernier ; PTad ajusté : poids du tissu adipeux périrénal ajusté pour le poids de la carcasse. 
II apparaît une interaction significative entre la taille de portée d'origine et le type génétique du père sur la proportion de l'arrière de la carcasse et son adiposité. Pour ces caractères, les coefficients de régression relatifs au type paternel 9077 sont significativement différents de 0 , contrairement à ceux des 2 autres types. Chez les produits de ce type génétique, une augmentation de la taille de portée se traduit par une réduction de l'arrière de la carcasse et une augmentation de l'adiposité.

\section{Paramètres du croisement (tableau V)}

\section{Effets génétiques additifs}

Le poids à $30 \mathrm{j}$ dépend des effets maternels mais aussi d'effets génétiques directs.
On oberve une opposition entre l'effet maternel favorable de la souche 9077 et celui, défavorable, des souches sélectionnées sur la taille de portée. Les effets directs sont prépondérants sur la vitesse de croissance entre 30 et $79 j$ et sur le poids à $79 j$. A l'opposé de la souche 1066, la souche 9077 exerce l'effet direct le plus favorable sur la vitesse de croissance et le poids à 79 j. Entre souches, il apparaît une opposition entre les effets directs sur la vitesse de croissance et les effets directs sur le poids de carcasse ajusté ; la souche 9077 exerce les effets directs les plus favorables sur la vitesse de croissance et les plus défavorables sur le poids de carcasse ajusté ; c'est l'inverse pour la souche 1066. La proportion de l'arrière de la carcasse est augmentée par les effets directs de la souche 1066 et les effets maternels de la souche

Tableau V. Paramètres de croisement des caractères de croissance et de carcasse.

\begin{tabular}{|c|c|c|c|c|c|c|c|}
\hline & & $P 30(g)$ & $P 79(g)$ & $D P(g)$ & $\begin{array}{c}P C a r \\
\text { ajusté }(g)\end{array}$ & $\begin{array}{c}\text { PMpos } \\
\text { ajusté }(g)\end{array}$ & $\begin{array}{c}\text { PTad } \\
\text { ajusté }(g)\end{array}$ \\
\hline$g^{\prime}$ & $\begin{array}{l}9077 \\
1066 \\
1077 \\
\sigma g^{\prime}\end{array}$ & $\begin{array}{c}-7^{a b} \\
24^{a} \\
-17^{b} \\
12\end{array}$ & $\begin{array}{c}96^{\mathrm{a}} \\
-68^{\mathrm{b}} \\
-28^{\mathrm{b}} \\
24\end{array}$ & $\begin{array}{c}103^{a} \\
-92^{c} \\
-11^{b} \\
19\end{array}$ & $\begin{array}{r}-16 c \\
15^{a} \\
1 b \\
7\end{array}$ & $\begin{array}{c}-3 b \\
6 a \\
-30 \\
1\end{array}$ & $\begin{array}{c}3 a \\
-10 \\
-20 \\
1\end{array}$ \\
\hline$g^{M}$ & $\begin{array}{l}9077 \\
1066 \\
1077 \\
\sigma g^{M}\end{array}$ & $\begin{array}{c}40^{a} \\
-24^{\circ} \\
-16^{b} \\
8\end{array}$ & $\begin{array}{c}45^{a} \\
10^{a} \\
-55^{b} \\
16\end{array}$ & $\begin{array}{r}5 \mathrm{~b} \\
34^{\mathrm{a}} \\
-39^{\mathrm{c}} \\
12\end{array}$ & $\begin{array}{c}-2^{b} \\
6^{a} \\
-4{ }^{b} \\
5\end{array}$ & $\begin{array}{l}4 a \\
-2 b \\
-1 b \\
i\end{array}$ & $\begin{array}{c}-1,4^{\mathrm{b}} \\
0,8 \mathrm{a} \\
0,6^{\mathrm{a}} \\
0,6\end{array}$ \\
\hline$h^{\prime}$ & $\begin{array}{l}1066 \times 1077 \\
1077 \times 9077 \\
9077 \times 1066 \\
h^{\prime} \text { moyen }\end{array}$ & $\begin{array}{l}4^{*} \\
6 * \\
4^{*} \\
5\end{array}$ & $\begin{array}{l}6 * \\
2 \\
2 \\
3\end{array}$ & $\begin{array}{l}7^{*} \\
1 \\
1 \\
3\end{array}$ & $\begin{array}{l}0 \\
2^{*} \\
2^{*} \\
2\end{array}$ & $\begin{array}{l}0 \\
0 \\
1 \\
0\end{array}$ & $\begin{array}{c}4 \\
-9 \\
0 \\
-2\end{array}$ \\
\hline
\end{tabular}

$g^{\prime}$ : effets génétiques additifs directs ; $g^{M}$ : somme des effets génétiques additifs maternels et grands matemels ; og : écart-type des estimées des effets génétiques additifs; $h^{h}=$ hétérosis direct, en pourcentage de la moyenne parentale ; * : significativement différent de $0(P<0,05)$. Les estimées $\mathrm{g}^{\prime}$ et $\mathrm{g}^{\mathrm{M}}$ affectées de lettres différentes sont significativement differentes $(P<0,05)$. P30 : poids vif à $30 j ; P 79$ : poids vif à $79 \mathrm{j}$; DP : gain de poids entre 30 et $79 \mathrm{j}$; PCar ajusté : poids de carcasse commerciale ajusté pour le poids vif à $79 \mathrm{j}$; MPpos ajusté : poids d'un membre postérieur ajusté pour le poids de carcasse ; PFrCo ajusté : poids de la fraction comestible d’un membre postérieur ajusté pour le poids de ce demier ; PTad ajusté : poids du tissu adipeux périrénal ajusté pour le poids de la carcasse. 
9077. Cette dernière exerce des effets directs qui augmentent l'adiposité et des effets maternels qui la diminuent, à l'inverse des 2 autres souches.

\section{Effets d'hétérosis}

Quel que soit le croisement, des effets d'hétérosis significatifs sont observés sur le poids à $30 \mathrm{j}$ (de l'ordre de $5 \%$ ) et sur le poids de carcasse ajusté (de l'ordre de $2 \%$ ). Dans le croisement des souches 1066 et 1077 un effet d'hétérosis est également observé sur le poids à 79 j et la vitesse de croissance. Le croisement des souches 1077 et 9077 entraîne un effet d'hétérosis qui diminue l'adiposité de la carcasse.

\section{Effet de la sélection dans la souche 1077}

L'effet de la sélection a été estimé, dans un premier temps, en comparant les performances des lapereaux des souches sélectionnée (1077) et témoin (9077) (tableau III). Dans un deuxième temps, cet effet global a été analysé en ses composantes directes et maternelles selon le modèle de Dickerson (1969) (tableau VI).
Les lapereaux de souche 1077 sont plus légers au sevrage ( 526 contre $592 \mathrm{~g}$ ), à $79 \mathrm{j}$ ( 2036 contre $2262 \mathrm{~g}$ ) et au stade adulte (3 860 contre $4130 \mathrm{~g}$ ) que ceux de la souche 9077 ; leur croissance pendant l'engraissement est moins rapide $(30,8$ contre $34,1 \mathrm{~g} / \mathrm{j}$ ). La sélection sur la prolificité a réduit le format et la vitesse de croissance, sous réserve que ces derniers caractères n'aient pas été augmentés, dans la souche témoin, par une sélection involontaire. Dans la logique de Taylor (1965), la souche 1077, la plus légère à l'état adulte, devrait présenter le degré de maturité pondérale le plus élevé à $79 \mathrm{j}$. Cela n'est pas le cas : la souche 1077 atteint à 79 j le plus faible pourcentage de son poids adulte $(52,7$ contre $54,8 \%$ ). Cette plus faible maturité pondérale est en cohérence avec un poids de tissu adipeux ajusté réduit (15,3 contre $19,0 \mathrm{~g}$ ) et un poids de membre postérieur ajusté inférieur (177,2 contre 181,8 g).

La sélection s'est traduite par une réduction des effets génétiques directs et maternels sur la vitesse de croissance et le poids à 79 j. Elle s'est également traduite par l'apparition d'effets maternels qui diminuent la proportion de l'arrière de la carcasse. Enfin, elle a induit des effets directs et mater-

Tableau VI. Effet de la sélection pour la prolificité dans la souche 1077 sur les composantes directe et maternelle de la valeur bouchère : écart entre la souche sélectionnée et la souche témoin 9077.

\begin{tabular}{lcccccc}
\hline Caractère & $P 30(g)$ & $P 79(g)$ & $D P(g)$ & $\begin{array}{c}P C a r \\
\text { ajusté }(g)\end{array}$ & $\begin{array}{c}P M p o s \\
\text { ajusté }(g)\end{array}$ & $\begin{array}{c}P T a d \\
\text { ajusté }(g)\end{array}$ \\
\hline$g_{1077}^{\prime}-g_{9077}^{\prime}$ & $-10 \pm 17$ & $-124 \pm 35^{*}$ & $-114 \pm 28^{*}$ & $17 \pm 12$ & $0 \pm 2$ & $-6 \pm 2^{*}$ \\
$g_{1077}^{\mathrm{M}}-g_{9077}^{\mathrm{M}}$ & $-56 \pm 11^{*}$ & $-100 \pm 23^{*}$ & $-44 \pm 18^{*}$ & $-2 \pm 8$ & $-5 \pm 1^{*}$ & $+2 \pm 1^{*}$ \\
$\mathrm{~g}_{1077}^{\top}-g_{9077}^{\top}$ & $-66 \pm 16^{*}$ & $-224 \pm 34^{*}$ & $-158 \pm 27^{*}$ & $15 \pm 10$ & $-5 \pm 2^{*}$ & $-4 \pm 1^{*}$ \\
\hline
\end{tabular}

\footnotetext{
* : valeur significativement différente de zéro $(P<0,05) ; g_{k}^{\prime}, g_{k}^{M}$ et $g_{k}^{\top}$ : effet génétique direct, maternel et global de la souche k. P30 : poids vif à $30 \mathrm{j}$; P79 : poids vif à $79 \mathrm{j}$; DP : gain de poids entre 30 et $79 \mathrm{j}$; PCar ajusté : poids de carcasse commerciale ajusté pour le poids vif à $79 \mathrm{j}$; MPpos ajusté : poids d'un membre postérieur ajusté pour le poids de carcasse ; PFrCo ajusté : poids de la fraction comestible d'un membre postérieur ajusté pour le poids de ce dernier ; PTad ajusté : poids du tissu adipeux périrénal ajusté pour le poids de la carcasse.
} 
nels, respectivement négatifs et positifs, sur l'adiposité.

\section{DISCUSSION}

Les présentes estimations des paramètres du croisement, à l'exception des effets maternels sur la vitesse de croissance, concordent avec la plupart des estimées antérieures (Brun et Ouhayoun, 1989), bien que ces dernières n'aient pas été ajustées pour la taille de portée au sevrage.

Il est intéressant de rapprocher les paramètres de la croissance estimés dans la présente étude et les paramètres de la taille de portée estimés par Bolet et al (1990) et par Brun (1990, 1993), sur les mêmes souches. Le classement des souches 9077 , 1066 et 1077 pour l'effet maternel sur le poids à $30 \mathrm{j}(40 \mathrm{~g},-24 \mathrm{~g},-16 \mathrm{~g}$, respectivement) est l'inverse de celui qui est observé sur la taille de portée ou ses composantes $(-0,7,0,5$ et 0,2 lapereaux nés totaux et $-0,6,0,4$ et 0,2 sites d'implantation). Entre les 3 souches étudiées, les effets maternels sur le poids à $30 \mathrm{j}$ apparaissent ainsi largement déterminés par les différences de taille de portée entre ces souches.

De même, les effets directs sur la croissance postsevrage estimés ici ( $103 \mathrm{~g},-92 \mathrm{~g}$ et $-11 \mathrm{~g}$, respectivement) se classent comme les effets directs sur la taille de la portée à la naissance $(0,4,-0,4$ et 0,0 lapereau). Étant donné que les effets directs sur la taille de portée sont déterminés par les gènes de viabilité des fotus, le potentiel de croissance des lapereaux apparaît comme un facteur de viabilité.

Pour le développement relatif de l'arrière et l'adiposité, les effets maternels des souches peuvent s'expliquer par l'effet de la taille de portée au sevrage. Ainsi, l'effet maternel positif sur le développement relatif de l'arrière et négatif sur l'adiposité de la souche 9077 peut s'expliquer par le signe de la liaison entre la taille de portée et ces caractères. Ce n'est plus le cas pour le poids à $79 \mathrm{j}$ et la vitesse de croissance, sans doute à cause des effets d'interaction entre taille de portée et type génétique de mère.

Les relations d'allométrie de croissance des tissus et des organes existant intrasouche (Cantier et al, 1969) suggéraient une liaison positive entre le rendement en carcasse, le développement relatif du membre postérieur et l'adiposité de la carcasse, 3 expressions de la maturité corporelle. Cela n'est que partiellement vérifié. Ainsi, la souche 1066, qui présente à la fois le meilleur rendement et le plus grand développement de l'arrière, n'a pas la plus forte adiposité. Dans la comparaison des souches pures 9077 et 1077 , la première, qui présente la plus forte adiposité et le plus fort développement de l'arrière n'a pas le meilleur rendement. De même, en ce qui concerne les effets directs, par exemple, la souche 9077 favorise l'adiposité, alors qu'elle diminue le rendement et le développement de l'arrière. Cela confirme des observations antérieures (Ouhayoun, 1978), selon lesquelles les critères de maturité corporelle manifestent une certaine indépendance lorsqu'on se situe, non pas intrasouche mais en comparaison de souches.

Chez le lapin, les expériences de sélection sur la prolificité faisant état de réponses corrélées sur la croissance et la composition corporelle sont rares (Rochambeau, 1988). On s'y limite généralement à la réponse sur le poids au sevrage (Matheron et Poujardieu, 1984 ; Mgheni et Christensen, 1985 ; Narayan et al, 1985). Les seules références existant chez le lapin concernent 2 souches expérimentales sélectionnées en Espagne (Camacho et Baselga, 1990 ; Estany et al, 1989). Les réponses sur la composition corporelle ne sont pas significatives. Sur la croissance, elles diffèrent selon la souche: les corrélations génétiques réalisées entre taille de portée au sevrage et vitesse de croissance $28-77$ j sont de 0,42 et $-0,65$. 
Cette dernière valeur indiquant un antagonisme génétique entre taille de portée et vitesse de croissance est conforme à celle observée dans la souche 1077.

L'influence de la taille de portée d'origine sur le poids à 30 et à 79 j confirme des résultats antérieurs (Rouvier et al, 1973). En revanche, cette influence sur le gain de poids est un résultat original.

L'existence d'effet de la taille de portée d'origine et d'interactions types génétiques $x$ taille de portée sur les caractères de croissance conduit, d'une part, à relativiser les estimées des valeurs moyennes des types génétiques des lapereaux et, par conséquent, les paramètres du croisement, d'autre part, à prendre en compte la taille de portée dans la planification d'expériences visant à l'évaluation génétique des caractères de croissance.

Le poids au sevrage des lapereaux de père 1077 n'est pas diminué par une augmentation de la taille de portée. Cela peut être dû au fait que leur faible potentiel de poids à $30 \mathrm{j}$, estimé par ce poids exprimé dans des conditions non limitantes (portées peu nombreuses), les rend moins sensibles aux limites nutritionnelles imposées par la mère. Le potentiel de poids de ces lapereaux est, en effet, significativement inférieur à celui des lapereaux des autres types génétiques de père (tableau VII).

Les lapereaux de mère 1066 et, dans une moindre mesure, les lapereaux de père de ce type génétique se révèlent incapables

Tableau VII. Poids à $30 \mathrm{j}$ selon le type de père.

\begin{tabular}{llll}
\hline & \multicolumn{3}{c}{ Type de père } \\
& 9077 & 1066 & 1077 \\
& & & \\
\hline Portée de 4 à 8 & $626^{\mathrm{a}}$ & $644^{\mathrm{a}}$ & $581^{\mathrm{b}}$ \\
Portée de 5 à 13 & $538^{\mathrm{b}}$ & $544^{\mathrm{b}}$ & $557^{\mathrm{b}}$ \\
\hline
\end{tabular}

Intra type de père, les valeurs affectées de lettres différentes $(P<0,05)$ sont significativement différentes. de compenser le déficit pondéral à 30 j résultant d'une taille de portée élevée. Cette inaptitude à la croissance compensatrice pourrait résulter d'effets génétiques à la fois directs et matemels, propres à cette souche.

L'aptitude à croître dans une portée nombreuse, qui peut être considérée comme un milieu difficile, relève du phénomène général de la "résilience». La présente étude met clairement en évidence une variation génétique de ce caractère mais les effectifs réduits de cette expérience ne permettent pas de l'analyser plus précisément.

\section{CONCLUSION}

Cette expérience de croisement entre 3 souches de lapin de format adulte moyen confirme l'existence de différences significatives pour les paramètres du croisement des caractères de croissance et de composition corporelle.

La souche 9077 présente des effets directs et maternels favorables sur la vitesse de croissance et le poids à l'abattage (79 j) mais des effets directs défavorables sur les qualités bouchères (rendement en carcasse, développement de l'arrière, adiposité). La souche 1066 exerce des effets directs défavorables sur le poids à 79 j mais favorables sur les qualités bouchères. Enfin, la souche 1077 exerce des effets maternels défavorables sur le poids à 79 j et sur le rendement en carcasse.

Apparemment, la sélection sur la prolificité dans la race Néo-Zélandaise s'est traduite par une diminution de la croissance, de la proportion de l'arrière de la carcasse et de l'adiposité.

La mise en évidence d'interactions entre les tailles de portée et les effets d'origine génétique, comme par exemple le type génétique du lapereau, ouvre une voie de recherche nouvelle chez le lapin : analyse génétique de la résistance des caractères 
pondéraux à une augmentation de la taille de portée d'origine, un aspect du phénomène général de la résilience.

\section{REMERCIEMENTS}

Les auteurs remercient $D$ Delmas, pour sa contribution à cette expérimentation, le personnel technique des 2 stations, en particulier $Y$ Andreuzza, $\mathrm{J}$ Falières et $\mathrm{V}$ Tartié, ainsi que les lecteurs du manuscrit pour leurs critiques constructives.

\section{REFERENCES}

Blasco A, Ouhayoun J, Masoero G (1993) Harmonization of criteria and terminology in rabbit meat research. World Rabbit Science 1, 3-10

Bolet $G$, Brun JM, Hulot F, Theau-Clément M (1990) Variabilité génétique et effet de la sélection dans le croisement de trois souches de lapin. II. Composantes biologiques de la taille de portée. In : $5^{e s} J$ Rech Cunic, Paris, comm 65, ITAVI, Paris

Brun JM (1990) Variabilité génétique et effet de la sélection dans le croisement de trois souches de lapin. I. Caractères des portées à la naissance et au sevrage. In : $5^{\ominus s} \mathrm{~J}$ Rech Cunic, Paris, comm 64, ITAVI, Paris

Brun JM (1993) Paramètres du croisement entre trois souches de lapin et analyse de la réponse de la sélection sur la taille de portée. Génét Sél Evol 25, 459-474

Brun JM, Ouhayoun J (1989) Growth performances and carcass traits in three strains of rabbits on their two-way crosses. Ann Zootech 38, 171-179

Brun JM, Rouvier R (1984) Effets génétiques sur les caractères des portées issues de trois souches de lapin utilisées en croisement. Génét Sél Evol 16, 367-384
Camacho J, Baselga M (1990) Estimation des corrélations génétiques entre caractères de reproduction et de croissance à travers la réponse à la sélection. In: $5^{\ominus s} \mathrm{~J}$ Rech Cunic, Paris, comm 66, Itavi, Paris

Cantier J, Vezinhet A, Rouvier R, Dauzier L (1969) Allométrie de croissance chez le lapin (Oryctolagus cuniculus). I. Principaux organes et tissus. Ann Biol Anim Bioch Biophys 9, 5-39

Dickerson GE (1969) Experimental approaches in utilizing breed resources. Anim Breed Abstr 37, 191-202

Estany J, Baselga M, Blasco A, Camacho J (1989) Mixed model methodology for the estimation of genetic response to selection for litter size of rabbits. Livest Prod Sci 21, 61-75

Matheron G, Poujardieu B (1984) Expérience de sélection de la taille de portée chez la lapine. In: I/Ith World Rabbit Congress, Roma, 3-34, World Rabbit Science Association

Mgheni M, Christensen K (1985) Selection experiments on growth and litter size in rabbits. Acta Agr Scand 35, 278-294

Narayan AD, Rawat S, Saxena MC (1985) Evaluation of response to selection for litter size in rabbits. Indian J Anim Sci 55, 954-957

Ouhayoun J (1978) Étude comparative de races de lapin différant par le poids adulte. Incidence du format paternel sur les composantes de la croissance des lapereaux issus de croisement terminal. Thèse Univ Sci Techn Languedoc, Montpellier, $72 p+$ annexes

Rochambeau $H$ de (1988) Genetics of the rabbit for wool and meat production (1984-1987). In: IVth World Rabbit Congress, Budapest, 1 68, World Rabbit Science Association

Rouvier R, Poujardieu B, Vrillon JL (1973) Analyse statistique des performances d'élevage des lapines. Facteurs du milieu, corrélations, répétabilités. Ann Génét Sél Anim 5, 83-107

SAS/Stat (1990) User's guide, Version 6, Cary, NC

Taylor SCS (1965) A relation between mature weight and time taken to mature in animals. Anim Prod 1, 203-220 